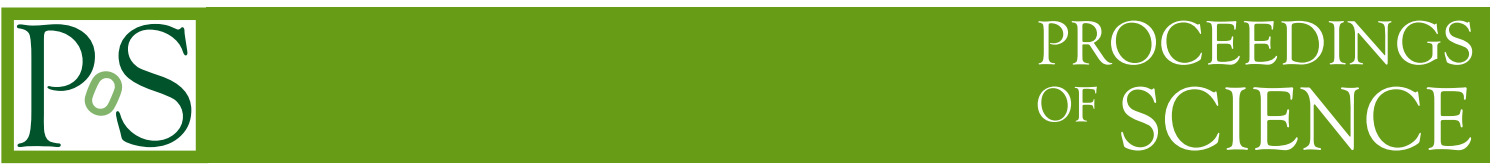

\title{
The Early Career, Gender and Diversity office at LHCb
}

\author{
Eluned Smith, on behalf of the LHCb collaboration ${ }^{a, *}$ \\ ${ }^{a}$ RWTH, Aachen \\ E-mail: eluned.anne.smith@cern.ch
}

$\mathrm{LHCb}$ is a collaboration of over 1300 members from 83 institutions based in 19 countries, and representing many more nationalities. We aim to work together on experimental high energy physics, and to do so in the best and most collaborative conditions. The Early Career, Gender \& Diversity (ECGD) office exists to support this goal, and in particular has a mandate to support early-career physicists, to work towards gender equality, and support diversity in the collaboration. The ECGD officers advise the LHCb management and act as LHCb contacts for all matters related to ECGD. They are available for listening to and advising - in a confidential manner - colleagues who have witnessed or have been subject to harassment, discrimination or other inappropriate behaviour. They help raise awareness in the collaboration for topics related to ECGD. In this talk we briefly introduce the ECGD office, discuss what we have learnt from analysis of the collaboration's demographics and responses from a survey that we conducted recently, share our experience gained over the last years, and we present our vision for the future evolution of the ECGD.

40th International Conference on High Energy physics - ICHEP2020

July 28 - August 6, 2020

Prague, Czech Republic (virtual meeting)

\footnotetext{
${ }^{*}$ Speaker
} 


\section{The early career gender and diversity (ECGD) office at LHCb}

The early career gender and diversity office at $\mathrm{LHCb}$ consists of two officers who serve a two year period. It was founded in 2014 and its role is defined in the LHCb constitution. The aim of the ECGD office is to oversee the well-being and working environment of all LHCb members. The officers provide pastoral support to LHCb members, listening to and advising members who feel they have been subjected to harassment or discrimination, or who are suffering for other reasons. Officers may use their role to informally intervene in situations where it is felt harassment has occurred, but they have no hard power. One of the officers also attends collaboration board meetings, although does not have a vote. ECGD officers are typically senior collaboration members, but there are currently ongoing plans to add an additional two officers who would explicitly represent the early career community. There are ECGD open meetings four times a year, where matters related to ECGD issues are discussed publicly within the collaboration. An additional role of ECGD officers is to collate regular statistics and other relevant information to monitor progress related to gender and other protected characteristics, such as age, disability, family circumstances, sex, ethnicity and religion. Surveys were first carried out in 2013, and have been carried out in 2016 and 2019 subsequently. A selection of the results of the 2019 survey was the main focus of this talk, and is summarised in the following sections. These results are only collected on a sub-sample of LHCb members who voluntarily filled in the survey, thus one should be aware of potential selection bias effects. Nonetheless, the general trends are insightful and may be relevant for other particle physics experiments.

\section{Summary of some key results from the 2019 survey}

Around $120 \mathrm{LHCb}$ members ( $\sim 15 \%$ of active physicists) took part in the survey, with women much more likely to take part than men. Senior members were also proportionately slightly overrepresented. The survey consisted of multiple choice questions, with additional open-ended fields for comments. The survey was advertised via the general LHCb mailing list, and filling it out was voluntary. Given the over-representation of women amongst the respondents, results in the following are often quoted categorised according to gender. When quoting results in terms of gender, only those who identified as either male or female are compared, due to the reduced statistics for members who identify as non-binary.

\subsection{Work-life balance}

A total of $15 \%$ of respondents found their work like balance very unreasonable, and $57 \%$ found the balance very reasonable. Members with permanent positions were less likely to find their work-life balance reasonable ( $47 \%$ ), compared to post-docs $(65 \%)$. Women were less likely to find work-life balance compared to the men ( $44 \%$ vs $63 \%)$. General negative comments from the openended entries included feelings of guilt to go on holiday, detrimental effects on relationships and feelings of isolation, particularly when working abroad. Positive comments included the flexibility of work, and working long hours out of choice. 


\subsection{Impact of family on work}

Members were asked to assess the impact of family on their roles in LHCb. It was found that $35 \%$ of people at $\mathrm{LHCb}$ have caring responsibilities or dependants. Of this subset, $28 \%$ have either stepped down from or declined to accept a position of responsibility because of these responsibilities. There was a gender disparity, with $35 \%$ of women pertaining to this subset stepping down, in comparison with $20 \%$ of men. Gender differences were larger still when members were asked if they have taken some form of paternity, maternity or long term leave to care for a relative, with $32 \%$ of women, compared to $7 \%$ of men, saying they had. The gender disparity on whether said leave had a negative effect on their career was most stark, with $60 \%$ of women saying they were negatively affected, and no men. It was also found that women were more likely to move location due to their partner's job. General comments on family life included requests for more flexibility towards part-time work, and a normalisation of equal paternity and maternity leaves.

\subsection{Harassment, discrimination and disclosure}

Regarding disclosure of sexual orientation, $6.4 \%$ of respondents felt uncomfortable disclosing their sexual orientation at work. Respondents were also asked if they had ever experienced any kind of discrimination in their work environment, based on gender, ethnic origin, sexual identity, sexual orientation, disability, body shape, health (including mental health), age, or any other reason. In total $15 \%$ of respondents said they had, with $31 \%$ of women saying they had and $7 \%$ of men. The level of harassment is comparable with results of the 2016 survey. Further, $48 \%$ of women and $21 \%$ of men responded that they had been in situations where they felt uncomfortable or excluded because of inappropriate language/behaviour by their colleagues. When asked about disclosure of mental or physical health problems at work, $24 \%$ of respondents said they were uncomfortable doing so.

\subsection{Career advancement and mentoring}

The majority of respondents wanted to stay in academia. Those who do not plan to stay cite lack of long-term jobs, the two-body problem ${ }^{1}$, high levels of stress and poor remuneration as key reasons for not wishing to pursue an academic career further. Regarding uptake of a formal mentoring scheme, this was not very prevalent amongst members. To address this issue, the LHCb ECGD office, along with initiatives from other experiments, have founded the LHC Early Career mentorship scheme, which started running this autumn.

\subsection{Diversity training}

Respondents were asked if they had ever attended any gender and diversity related training. In total, $34 \%$ of respondents report they have attended such trainings, with $86 \%$ of saying attendance was compulsory. Of those that have attended such trainings, $38 \%$ said their actions and attitudes changed after training. Useful outputs from these trainings included a heightened awareness of unconscious bias, and the impact of language used in the work place.

\footnotetext{
${ }^{1}$ the problem of both life partners being in academia, resulting in difficulty for both partners to find academic jobs within a commutable distance
} 


\subsection{Conclusions}

The LHCb ECGD office aims to improve the working experience of members. It was founded in 2014 and is still evolving. One of the duties of the office is to collate data from LHCb members through the form of surveys to monitor progress related to gender and other protected characteristics. These proceedings outlined some of the key results from the 2019 survey. 